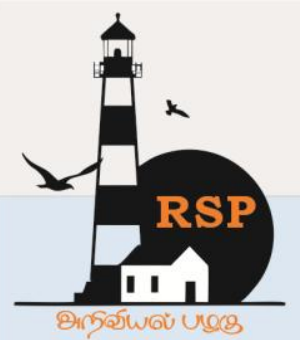

INTERNATIONAL RESEARCH JOURNAL ON ADVANCED SCIENCE HUB ISSN : $2582-4376$
Open Access

RSP SCIENCE HUB

(The Hub of Research Ideas)

Available online at www.rspsciencehub.com

\title{
Flow of Electrically Conducting Williamson Fluid with Cattaneo-Christov heat flux due to Permeable Sheet
}

Atul Kumar Ray ${ }^{1}$

${ }^{l}$ Assistant Professor of Mathematics, Department of Basic Science and Humanities, Government College of Engineering Keonjhar, Odisha-758002, India

atulkr@gcekjr.ac.in ${ }^{1}$

\begin{abstract}
An analysis is presented for the flow of non-Newtonian Williamson fluid due to permeable sheet. The recent modification of Fourier's law of heat conduction, i.e. Model of Cattaneo-Christov heat flux is studied in present article to analysis the heat flux. The permeable sheet undergoes suction and the effect of Soret and Dufour is also investigated The governing boundary layer equations are transformed into a non-dimensional form using appropriate set of transformation and the resulting non-linear coupled system of ordinary differential equations are solved using robust Homotopy Analysis method. The influence of physical parameter affecting the velocity of fluid, heat and mass transfer are discussed and outputs are expressed in figures and tables. Some interesting results are found from the study that the velocity profile is decelerating with increase in magnetic parameter. Increasing Darcy number accelerates the flow due to a corresponding rise in permeability of the regime and concomitant decrease in Darcian impedance.
\end{abstract}

Keywords: Williamson fluid, Cattaneo-Christov Heat Flux Model, Homotopy Analysis Method, Electrically Conducting Fluid, Permeable Sheet

\section{Introduction}

The electrically conducting fluid flow in the presence of magnetic field (MagnetoHydrodynamics) over permeable stretching sheet has importance and applications in heat exchanger, metal-working process in hot rolling, cooling process of nuclear reactor containment vessel and petrochemical industry. Crane [1] had provided analytical solution for flow of incompressible Newtonian fluid over a stretching sheet and the study of magneto-hydrodynamic (MHD) was first done by Alfven [2]. By incorporating MHD effect, the impact of boundary layer flow due to stretching sheet can be improved by applying magnetic field normal to the plane of the sheet which has the electrically conducting fluid. Further, the flow of electrical conducting Newtonian fluid in presence of uniform magnetic field between two parallel plates is analysed by Hartman and Lazarus [3]. Kumaran et al. [4] analyzed the electrically conducting fluid flow past a stretching permeable sheet. Nayak et al. [5] investigated the natural convective due to flow of nanofluid over permeable sheet with thermal radiation in presence of magnetic field. Recently, Khashi'ie et al. [6] inspected the influence of stratification on MHD stagnation point flow past a stretching surface embedded in a porous medium.

Heat transfer phenomenon in diverse practical applicants used to predict by the empirical formula of Fourier's law [7]. However, Fourier's model has certain short comes that any disturbance felt instantly spread by whole material. To overcome this short comes of Fourier's model, Cattaneo [8] modified the Fourier's model by adding thermal relaxation time for heat flux. Further, Christov [9] used Oldroyds derivative in order to preserve material invariance in Cattaneo's model. Many 


\section{www.rspsciencehub.com}

researchers have used Cattaneo-Christov model to analyse the heat flux. Recently, Vasu et al. [10] examined the natural convectional flow of Jeffrey nanofluid by Cattaneo-Christov model past vertical plate. Reddy et al. [11] have studied influence of Cattaneo-Christov model for hybrid dusty fluid flow.

The applications of non-Newtonian flow are well known in science and engineering especially in field of bioscience (blood flow, stenosis) and industry like melts and solution of polymer with high molecular weights, emulsion coated sheets like photographic films, extrusion of plastic sheets etc. A single constitutive equation is not sufficient to explore the different behaviour of fluids. Many rheological models [12-14] have been developed time to time to overcome such limitation. The power law fluid [12], Spriggs fluid [13], YasudaCarreau fluid [12] and Powell-Eyring [14] etc. are some fluids which explain the properties of shear thinning and viscoelastic fluids. One of such fluid is Williamson fluid [15] which is shear thinning fluid. Williamson [15] proposed one of the shear thinning models to analyse the flow of shear thinning materials and verified the results experimentally. Many researchers have contributed valuable work to this field in past few years. Nadeem et al. [16] scrutinized the flow of Williamson fluid over stretching sheet. Kumar et al. [17] have analyzed the impact of non-uniform source of heat on the Williamson fluid flow past curved sheet.

Motivated by above discussed numerous applications, the present article probes the boundary layer flow of Williamson fluid induced by permeable stretching sheet in the presence of electro-magnetic field, chemical reaction, Soret and Dufour effects. Cattaneo-Christov heat flux model emphases the role of relaxation time and studies the heat transfer phenomenon. The focus is on the influence of magnetic field and thermal relaxation time on the flow velocity and temperature profiles. The governing equations are reduced to system of non-linear coupled ordinary differential equation by using suitable similarity transformation discussed in mathematical formulation section. Homotopy analysis method (HAM) [18] is used to analyse the flow behaviour of Williamson fluid due to permeable stretching sheet in presence of magnetic field. Results
Volume 02 Issue 12 December 2020

obtained are concluded in the Conclusion section.

\section{Mathematical Modeling}

Considering two-dimensional laminar and steady flow of Williamson fluid over the sheet $y=0$, provided that the flow is being restricted to $y>0$. In order to produce the stretching, equal and opposite forces are applied along $x$ axis. Origin is fixed. Heat transfer follows the Cattaneo-Christov model. The effect of Soret and Dufour is also considered. The equation which governs the flow generated by stretching sheet, that is continuity, momentum, energy and concentration equations, are given by

$$
\begin{gathered}
\frac{\partial u}{\partial x}+\frac{\partial v}{\partial y}=0 \\
u \frac{\partial u}{\partial x}+v \frac{\partial u}{\partial y}=v \frac{\partial^{2} u}{\partial y^{2}}+\sqrt{2} v \Gamma \frac{\partial u}{\partial y} \frac{\partial^{2} u}{\partial y^{2}}-\sigma B^{2} u-\frac{v}{\gamma_{1}} u \\
+g\left[\beta_{T}\left(T-T_{\infty}\right)+\beta_{C}\left(C-C_{\infty}\right)\right]
\end{gathered}
$$

$$
\begin{aligned}
& u \frac{\partial T}{\partial x}+v \frac{\partial T}{\partial y}+\lambda_{1}\left(\begin{array}{l}
u \frac{\partial u}{\partial x} \frac{\partial T}{\partial x}+v \frac{\partial v}{\partial y} \frac{\partial T}{\partial y}+u \frac{\partial v}{\partial x} \frac{\partial T}{\partial y} \\
+v \frac{\partial u}{\partial y} \frac{\partial T}{\partial x}+2 v u \frac{\partial^{2} T}{\partial x \partial y}+u^{2} \frac{\partial^{2} T}{\partial x^{2}}+v^{2} \frac{\partial^{2} T}{\partial y^{2}}
\end{array}\right) \\
& =\alpha \frac{\partial^{2} T}{\partial y^{2}}+\frac{D k_{T}}{c_{s} c_{p}} \frac{\partial^{2} C}{\partial y^{2}}
\end{aligned}
$$

$u \frac{\partial C}{\partial x}+v \frac{\partial C}{\partial y}=D \frac{\partial^{2} C}{\partial y^{2}}+\frac{D k_{T}}{T_{m}} \frac{\partial^{2} T}{\partial y^{2}}-k_{1}\left(C-C_{0}\right)$

And the boundary conditions are

$$
\begin{aligned}
& \text { At } y=0, u=u_{w}, v=v_{w}, T=T_{w}, C=C_{w} \\
& y \rightarrow \infty, u=0, \quad T=T_{\infty}, C=C_{\infty}
\end{aligned}
$$

whereu(x, y) and $\mathrm{v}(\mathrm{x}, \mathrm{y})$ are the velocity components along the flow direction and normal to the flow direction. Further, $\mathrm{T}$ is temperature, $\mathrm{T}_{\mathrm{w}}$ is the temperature at the wall of the sheet and $v$ is the kinematic viscosity. $\Gamma$ is fluid parameter. $v_{w}$ is mass flux velocity. Noting that if $v_{w}>0$ corresponds to injection while $v_{w}<0$ corresponds to suction. $\beta_{T}$ and $\beta_{C}$ are coefficient of thermal and 
concentration expansion. , $\alpha$ is the thermal diffusivity and $\mathrm{h}$ is the heat transfer coefficient. Introducing

$\psi=\left(v x u_{e}\right)^{1 / 2} f(\eta), \quad \eta=\left(\frac{u_{e}}{v x}\right)^{1 / 2} y, \quad \theta(\eta)=\frac{T-T_{\infty}}{T_{w}-T_{\infty}}$,

$\phi(\eta)=\frac{C-C_{\infty}}{C_{w}-C_{\infty}}$

where $\psi$ is the stream function defined as $u=\frac{\partial \psi}{\partial y}$ and $v=-\frac{\partial \psi}{\partial x}$ which identically satisfies continuity equation.. Thus, we have

$$
u=a x \frac{d f}{d \eta} \text { and } v=-(a x)^{1 / 2} f(\eta)
$$

where prime denotes differentiation with respect to $\eta$. After introducing (7), governing equations (1)(4) and corresponding boundary conditions, (5)-(6) are reduced to

$\frac{d^{3} f}{d \eta^{3}}+f \frac{d^{2} f}{d \eta^{2}}-\left(\frac{d f}{d \eta}\right)^{2}+\lambda \frac{d^{2} f}{d \eta^{2}} \frac{d^{3} f}{d \eta^{3}}-M \frac{d f}{d \eta}-\frac{1}{D_{a}} \frac{d f}{d \eta}+\Lambda(\theta+N \phi)=0$

$\frac{1}{\operatorname{Pr}} \frac{d^{2} \theta}{d \eta^{2}}+f \frac{d \theta}{d \eta}-\lambda_{1}\left(f \frac{d f}{d \eta} \frac{d \theta}{d \eta}+f^{2} \frac{d^{2} \theta}{d \eta^{2}}\right)+D_{f} \frac{d^{2} \phi}{d \eta^{2}}=0$

$$
\begin{aligned}
& \frac{1}{L e} \frac{d^{2} \phi}{d \eta^{2}}+f \frac{d \phi}{d \eta}+S r \frac{d^{2} \theta}{d \eta^{2}}-k_{1} \phi=0 \\
& f(0)=S,\left.\frac{d f}{d \eta}\right|_{\eta=0}=1 \theta(0)=1, \phi(0)=1 \\
& f(\infty)=0, \theta(\infty)=1, \phi(\infty)=0
\end{aligned}
$$

where prime denotes differentiation with respect to $\eta$, and material fluid parameter is represented by $W e, \quad$ Pris the Prandtl number. $\lambda$ are fluid parameters. $\lambda_{1}$ is thermal relaxation parameter. $D_{a}$,

$N=\frac{\beta_{c}\left(C_{w}-C_{\infty}\right)}{\beta_{T}\left(T_{w}-T_{\infty}\right)}, S r=\frac{D k_{T}\left(T_{w}-T_{\infty}\right)}{T_{m} \alpha\left(C_{w}-C_{\infty}\right)}, D_{f}=\frac{D k_{T}\left(C_{w}-C_{\infty}\right)}{c_{c} c_{p}\left(T_{w}-T_{\infty}\right)}$ and $S$ are respectively Dufour number,concentration buoyancy parameter, Soret number, Darcy and suction parameter.

The physical quantities of interest are the skin friction coefficient $C_{f}$ and the local Nusselt number $N u_{x}$ which are given by

$$
\begin{aligned}
C_{f} & =\frac{\tau_{w}}{\rho u_{e}{ }^{2}} \\
S h_{x} & =\frac{x m_{w}}{D\left(C_{w}-C_{\infty}\right)}
\end{aligned}
$$

After transformation, we have

$\tau_{w}=\left(\frac{\partial u}{\partial y}\right)_{y=0}, q_{w}=-\left(\frac{\partial \theta}{\partial y}\right)_{y=0}$ and $m_{w}=-\left(\frac{\partial \phi}{\partial y}\right)_{y=0}$

then dimensionless shear stress and rate of heat transfer and rate of mass transfer becomes

$$
\begin{aligned}
& \tau_{w}=\left.\left(\frac{d^{2} f}{d \eta^{2}}+\frac{\lambda}{2}\left(\frac{d^{2} f}{d \eta^{2}}\right)^{2}\right)\right|_{\eta=0}, \quad q_{w}=-\left.\frac{d \theta}{d \eta}\right|_{\eta=0} \text { and } \\
& m_{w}=-\left.\frac{d \phi}{d \eta}\right|_{\eta=0}
\end{aligned}
$$

\section{Methodology}

Homotopy analysis technique [18] is semi numerical strategy and utilized by many scientists and researchers. This strategy is applied in numerous fluid flow problems just as applied science and engineering. The governing equations (1)-(6) are transformed to non-dimensionalized form (9)-(13) by using transformation (7). The resultant system of differential equations (9)-(13) is finally solved via HAM. Now to obtain the series solution of (9) - (13) using HAM, we choose the appropriate initial guesses $f_{0}, \theta_{0}$ and $\phi_{0}$ of $f$, $\theta$ and $\phi$ in the following form

$f_{0}=S-1+e^{-\eta}, \theta_{0}=e^{-\eta}$ and $\phi_{0}=e^{-\eta}$

Symbolic computation software MATHEMATICA 9 is used for computation purpose. The HAM depends highly on convergence control parameter $\hbar$, we plot the $t$-curves to get the admissible range of $t_{1}, t_{2}$ and $t_{3}$ for $f, g$ and $h$ respectively shown in Fig. 1 and Fig. 2. Also Table 1 shows the comparison of present results with the previously published result [19] and found to be in good agreement. The appropriate range for the control parameters $h_{1}, h_{2}$ and $h_{3}$ are $-0.9<h_{1}<-0.28$, $-0.6<\hbar_{2}<-0.25$ and $-0.65<\hbar_{3} \leq-0.25$ at $16^{\text {th }}$ order of approximation for values

$$
L e=2, \kappa=0.55, N r=0.25, D a=2, K_{1}=0.2
$$


$\operatorname{Pr}=7, S r=0.25, \lambda_{1}=0.1, S=0.1, D_{f}=0.25$, $\lambda=0.5, \gamma=0.1, M=0.1$,

$$
-1.2
$$

$-0.7$

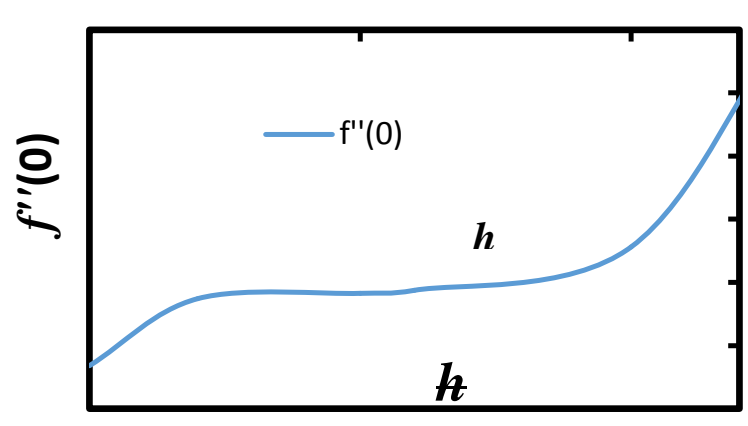

$-0.6$

$-0.8$

$-1$

$-1.2$

$-1.4$

$-1.6$

$-1.8$

Fig.1. $f "(0)$ vs. $\hbar$

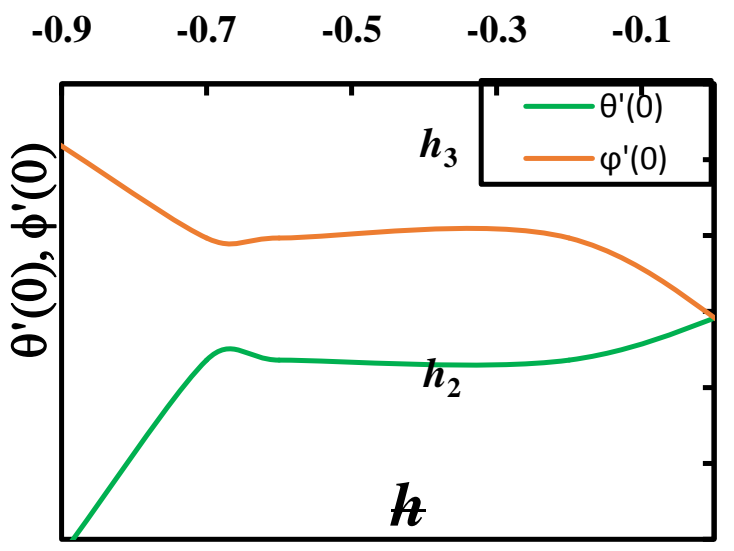

-0.6
-0.75
-0.9
-1.05
-1.2
-1.35
-1.5

Fig.2. $\theta^{\prime}(0)$ vs. $\hbar$ and $\phi^{\prime}(0)$ vs. $\hbar$

Table.1.Comparison of Present Result

\begin{tabular}{|c|c|c|}
\hline & \multicolumn{2}{|c|}{$-\tau_{w}$} \\
\hline$\lambda$ & Present & $\begin{array}{c}\text { Nadeem et al. } \\
{[19]}\end{array}$ \\
\hline 0 & 1.005045 & 1 \\
\hline 0.1 & 0.97041 & 0.976588 \\
\hline 0.2 & 0.93734 & 0.939817 \\
\hline 0.3 & 0.86541 & 0.88272 \\
\hline
\end{tabular}

\section{Result and Analysis}

The stream flow of an electrically leading Williamson fluid due to horizontal extending surface embedded in a porous medium has been considered with the impacts of the CattaneoChristov heat flux model, Soret and Dufour boundaries. Here an endeavour is made to separate an insightful answer for the exceptionally confounded and profoundly coupled arrangement of conditions. Fig. 3 depicts the variation of velocity with $\eta$ for different values of Hartman number (magnetic parameter). It is found that as the effect of magnetic field increases, the velocity of Williamson fluid fall down significantly. It is due to drag force produced by magnetic field to the electrically leading Williamson fluid which results in lower down the velocity of the fluid within the boundary layer.

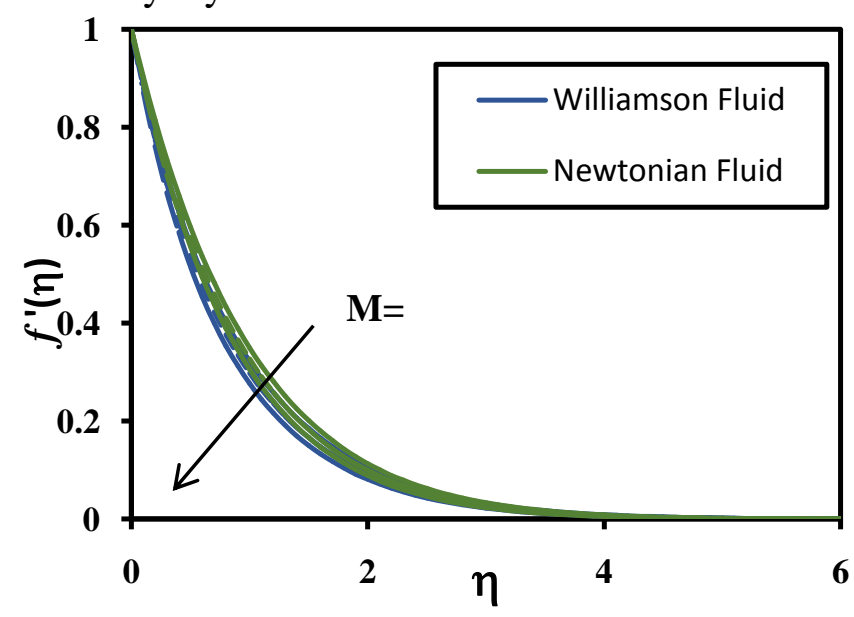

Fig.3.Variation of velocity for different $M$

The variation of temperature profile with $\eta$ for various values of MHD parameter is explored in Fig.4. It can be seen from the Fig. 4 that the thermal boundary layer increases with rise in MHD parameter. The influence of Lorentz force (drag force) reduced the velocity and generates friction on the flow which causes more heat energy. Due to this friction, temperature distribution in the flow field is increased. The behaviour of Williamson and Newtonian fluid are same for the effect of Hartman number on the profiles of velocity, temperature and concentration.

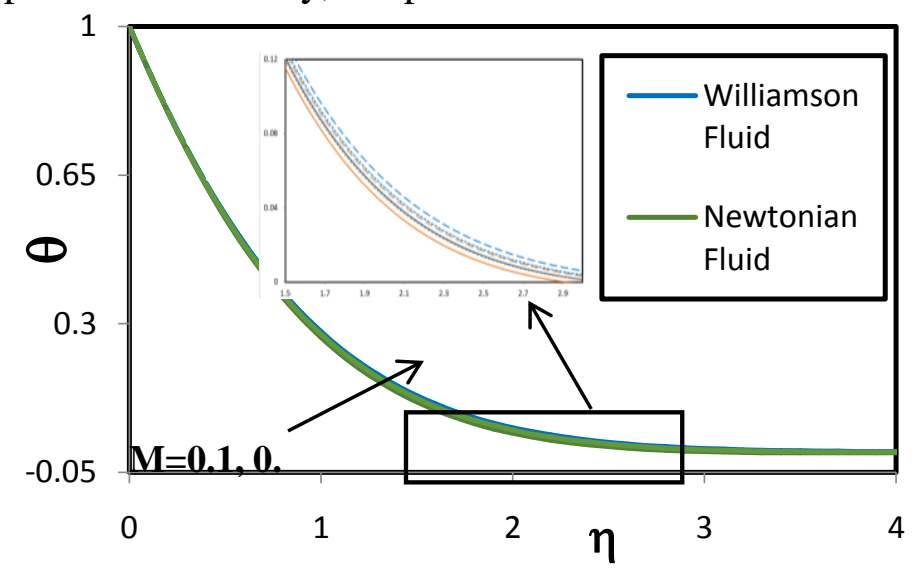

Fig. 4.Variation of temperature for different $M$ 
www.rspsciencehub.com

The effect of thermal relaxation parameter on the temperature field is explained in Fig. 5. $\lambda_{1}=0$ is corresponds to the Fourier's model. It is observed from the Fig. 5 that due to relaxation parameter, the temperature decreases. Also temperature for Cattaneo-Christov heat flux model is less than that of Fourier's model.

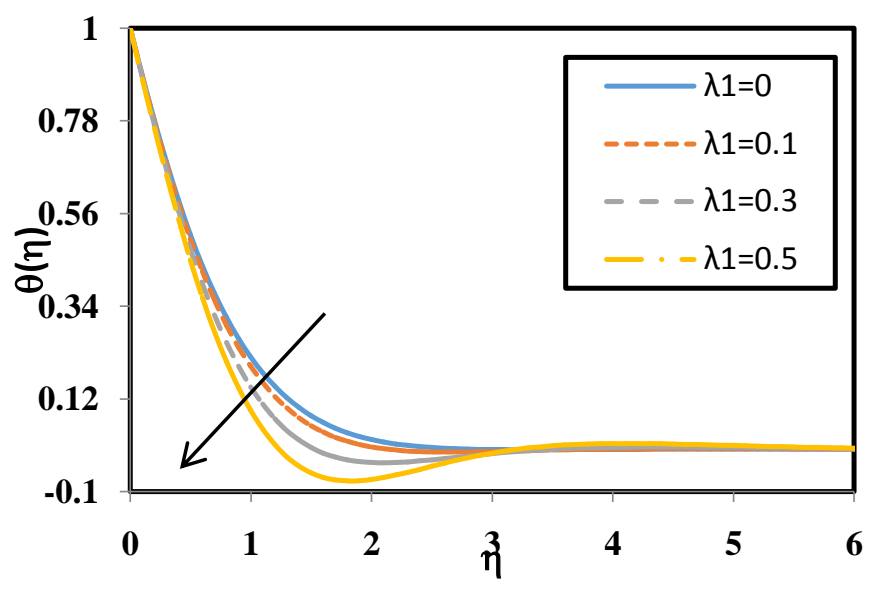

Fig. 5 Variation of temperature for different thermal relaxation parameter

\section{Conclusions}

The presented analysis leads to the following major outcomes:

- Convergence of the homotopy solution is discussed and the present results are compared with the existing results in published articles.

- Velocity profile is a reducing with magnetic parameter $M$ due to effect of Lorentz force whereas the temperature field is an increasing function of magnetic parameter $M$ because of friction between the wall of the sheet and fluid or within fluid causes heat generation.

- Temperature distribution is more in the Fourier's model for heat flux as compared to Cattaneo-Christov heat flux model.

- Temperature and thermal boundary layer thickness are decreasing functions of relaxation time

- The behaviours of parameters in CattaneoChristov model are qualitatively similar to those in Fourier's heat conduction law

\section{References}

[1] Crane, L. (1970). Flow past a stretching plate, ZeitshriftFürAngewandteMathematikundePhy sik $21645-647$
Volume 02 Issue 12 December 2020

[2] Alfven, H.

(1942).Existence

of electromagnetic-hydrodynamic waves, Nature 150, 405-406.

[3] Hartmann, J., \& Lazarus, F. (1937). Hgdynamics II. Theory of laminar flow of electrically conductive Liquids in a Homogeneous Magnetic Field.15(7).

[4] Kumaran, V., Banerjee, A. K., Kumar, A. V., \&Vajravelu, K. (2009). MHD flow past a stretching permeable sheet. Applied mathematics and computation.210(1), 26-32.

[5] Nayak, M. K., Akbar, N. S., Pandey, V. S., Khan, Z. H., \&Tripathi, D. (2017). 3D free convective MHD flow of nanofluid over permeable linear stretching sheet with thermal radiation. Powder Technology, 315, 205-215.

[6] Khashi'ie, N. S., Arifin, N. M., Rashidi, M. M., Hafidzuddin, E. H., \&Wahi, N. (2020). Magnetohydrodynamics (MHD) stagnation point flow past a shrinking/stretching surface with double stratification effect in a porous medium. Journal of Thermal Analysis and Calorimetry, 139(6), 3635-3648.

[7] Fourier, J. B. J. Theorieanalytique De La chaleur, Paris, (1822).

[8] Cattaneo, C. (1948). Sulla conduzionedelcalore. Atti Sem. Mat. Fis. Univ. Modena, 3, 83-101.

[9] Christov, C. I. (2009). On frame indifferent formulation of the Maxwell-Cattaneo model of finite-speed heat conduction. Mechanics Research Communications, 36(4), 481-486.

[10] Vasu, B., Ray, A. K., \& Gorla, R. S. (2020). Free convective heat transfer in Jeffrey fluid with suspended nanoparticles and CattaneoChristov heat flux. Proceedings of the Institution of Mechanical Engineers, Part N: Journal of Nanomaterials, Nanoengineering and Nanosystems, 2397791420912628.

[11] Reddy, M. G., Rani, M. S., Kumar, K. G., Prasannakumar, B. C., \&Lokesh, H. J. (2020). Hybrid dusty fluid flow through a CattaneoChristov heat flux model. Physica A: Statistical Mechanics and its Applications, 123975.

[12] Chhabra RP and Richardson JF. NonNewtonian flow and applied rheology: engineering applications. Oxford: Butterworth-Heinemann, (2011). 
[13] Ray, A. K., Vasu, B., \& Gorla, R. S. R. (2019). Homotopy simulation of nonNewtonian spriggs fluid flow over a flat plate with oscillating motion. International Journal of Applied Mechanics and Engineering, 24(2), 359-385.

[14] Ray, A. K., Vasu, B., Murthy, P. V. S. N., \& Gorla, R. S. (2020). Non-similar solution of Eyring-Powell fluid flow and heat transfer with convective boundary condition: Homotopy Analysis Method. International Journal of Applied and Computational Mathematics, 6(1), 16.

[15] Williamson, R. V. (1929). The flow of pseudoplastic materials.Industrial \& Engineering Chemistry Research, 21, No. 11, 1108.

[16] Nadeem, S., Hussain, S. T., \& Lee, C. (2013). Flow of a Williamson fluid over a stretching sheet. Brazilian journal of chemical engineering, 30(3), 619-625.

[17] Kumar, K. A., Reddy, J. R., Sugunamma, V., Sandeep, N. (2019). Simultaneous solutions for MHD flow of Williamson fluid over a curved sheet with nonuniform heat source/sink. Heat Transfer Research, 50(6).

[18] Liao, S. (2004). On the homotopy analysis method for nonlinear problems. Applied Mathematics and Computation, 147(2), 499513.

[19] Nadeem, S., Hussain, S. T., \& Lee, C. (2013). Flow of a Williamson fluid over a stretching sheet. Brazilian journal of chemical engineering, 30(3), 619-625. 